\title{
Intrusion-extrusion of electrolytic solutions in zeolites for energy storage
}

Rossella Arletti $^{1}$, Laura Ronchi ${ }^{2}$, Simona Quartieri ${ }^{3}$, Giovanna Vezzalini ${ }^{4}$, Andrey Ryzhikov ${ }^{2}$, Habiba Nouali ${ }^{2}$, Jean Daou ${ }^{2}$, Joel Patarin ${ }^{2}$

${ }^{1}$ Dipartimento Di Scienze Della Terra, Torino, Italy, ${ }^{2}$ Université de Strasbourg (UDS), Université de Haute Alsace (UHA), Equipe Matériaux à Porosité Contrôlée (MPC), Institut de Science des Matériaux de Mulhouse (IS2M), UMR CNRS 7361,, Mulhouse, F-68093, France, ${ }^{3}$ Dipartimento di Scienze Matematiche e Informatiche, Scienze Fisiche e Scienze della Terra, Università di Messina, Messina S. Agata, I-98166, Italy, ${ }^{4}$ Dipartimento di Scienze Chimiche e Geologiche, Università di Modena e Reggio Emilia, Modena, Modena, I41100, Italy

E-mail: rossella.arletti@unito.it

The demand for low cost, efficient and sustainable energy is ever increasing. Since fifteen years, absorption or energy storage using pure-silica zeolites (zeosils) has been developed and opened a new field of applications for these exciting microporous solids [1]. The basic idea focuses on the forced penetration (intrusion), upon a certain pressure, of water or aqueous salt solutions (non-wetting liquid) into a hydrophobic porous material. During this intrusion, the mechanical energy can be converted into interfacial one. Depending on various physical parameters of the porous material - such as its pore system, its dimensionality and pore size - when pressure is released (extrusion) the "zeosil-water" system is able to restore, dissipate or absorb the supplied mechanical energy accumulated during the compression step, with a more or less significant hysteresis, displaying a spring, shock-absorber or bumper behavior, respectively.

In the present work, the energetic performances of pure-silica zeolites (Si-FER and Si-CHA) are studied by means of intrusion-extrusion experiments with water and electrolytic aqueous solutions $(\mathrm{MgCl} 2, \mathrm{NaCl})$. The samples, before and after such experiments, have been fully characterized by conventional X-ray powder diffraction (XRPD), thermogravimetric (TG) analyses and N2 adsorption-desorption experiments. The intrusion of the species (water and cations) has been followed by situ high pressure (HP) synchrotron XRPD during compression and decompression. Diffraction experiments were carried out on the SNBL1 (BM01a) beamline at ESRF (Grenoble), using a modified Merril-Bassett DAC and the electrolytic aqueous solution as Pressure Transmitting Medium (PTM).

Two consecutive intrusion-extrusion cycles of $\mathrm{MgCl} 2 \bullet .21 \mathrm{H} 20$ on Si-FER were performed and the results were quite reproducible. The intrusion of $\mathrm{MgCl} 2$ electrolyte aqueous solution in Si-FER occurs in the range 150-250 $\mathrm{MPa}$. Once the pressure is released, the water is spontaneously expelled from the zeolite, and the intrusion/extrusion process is completely reversible thus "Si-FER-MgCl $2 \cdot 21 \mathrm{H} 2 \mathrm{O}$ "system displays a spring behavior. The structure refinement of the data collected at $0.28 \mathrm{GPa}$ (pressure close to the intrusion one) during compression indicated that both ions and water molecules penetrate the Si-FER channels. $0.8 \mathrm{Mg}, 2 \mathrm{Cl}$ atoms, and 7.8 water molecules per unit cell were located. Mg atoms are at the center of the FER cage, coordinating four partially occupied water molecules in a square planar configuration (Figure 1). The position of $\mathrm{Mg}$ cation perfectly matches that found by Alberti et al. [2] in natural ferrierite from Monastir (Sardinia - Italy). The Rietveld analysis supports an ideal composition of the intruded liquid close to $\mathrm{MgCl} 2 \cdot 10 \mathrm{H} 2 \mathrm{O}$. Interestingly, the composition of the intruded species differs from the initial solution, revealing a partial desolvation of both magnesium and chloride ions. For pressure values higher than $1.47 \mathrm{GPa}$ - as already observed for compression experiments of Si-FER with other PTMs - a phase transition to the monoclinic symmetry P21/n is observed [3].

Preliminary results on the intrusion of pure water and $\mathrm{NaCl}$ aqueous solutions at different concentrations in $\mathrm{Si}-\mathrm{CHA}$ will be presented.

[1] Eroshenko, V. et al. (2001). J. Am. Chem. Soc., 123, 8129.

[2] Alberti A. \& Sabelli C. (1987). Zeit. Krist., 178, 249.

[3] Arletti, R. et al. (2014). Micr. Mes. Mat. 191, 27.
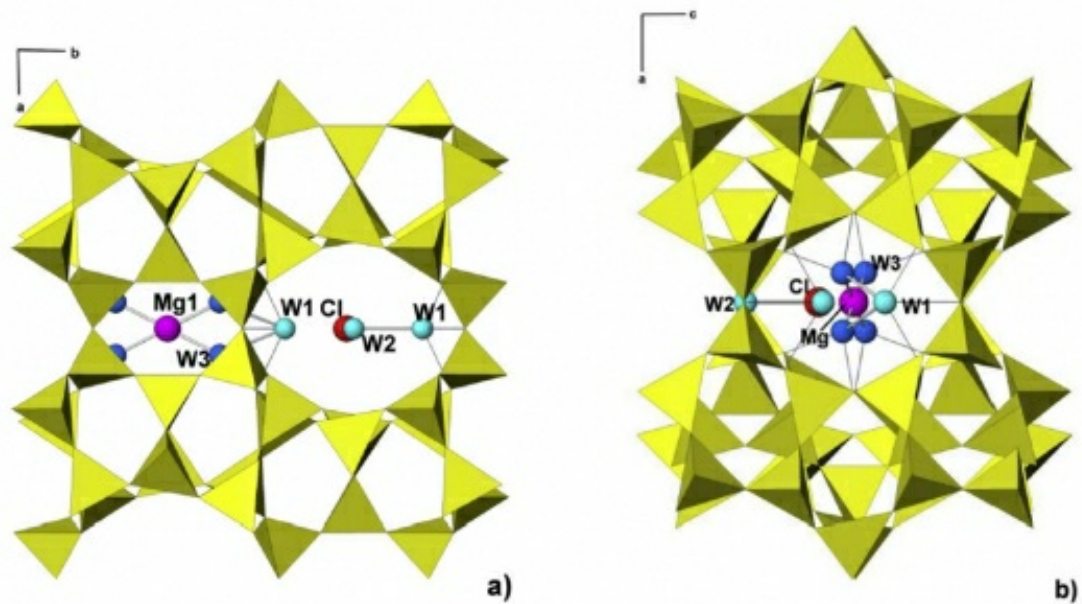

a)

Keywords: Zeolites, energy storage, in situ HP-XRPD 